\title{
Low-temperature solid-state FTIR study of glycine, sarcosine and $N, N$-dimethylglycine: observation of neutral forms of simple $\alpha$-amino acids in the solid state
}

\author{
A. Gómez-Zavaglia ${ }^{a b}$ and R. Fausto*a \\ ${ }^{a}$ Department of Chemistry, University of Coimbra, P-3004-535, Portugal \\ ${ }^{b}$ Facultad de Farmacia y Bioquímica, Universidad de Buenos Aires, RA-1113, Argentina. \\ E-mail: rfausto@ci.uc.pt
}

Received 30th April 2003, Accepted 19th June 2003
First published as an Advance Article on the web 4th July 2003

Neutral forms of glycine and their $N$-methylated derivatives, sarcosine ( $N$-methylglycine) and $N, N$ dimethylglycine were, for the first time, observed in the solid state pure compounds. The substances were sublimated under high vacuum, quickly deposited onto a cold CsI substrate at $9 \mathrm{~K}$ and examined using FTIR spectroscopy within the temperature range 9-300 K. For all the compounds studied, the spectra obtained at $9 \mathrm{~K}$ after deposition revealed the presence of both the neutral and zwitterionic amino acid forms. The identification of the neutral forms and their vibrational signatures could be unequivocally established by taking into consideration the available data for the matrix-isolated neutral monomeric and aggregated amino acids as well as solid state data for acid and alkaline salts of glycine. Upon temperature increase, neutral forms convert nonreversibly to the zwitterionic forms, as noticed by the systematic increase in the relative intensities of the infrared bands ascribable to the latter species. Observation of aggregates of neutral amino acids in the solid phase, together with the fact that the amount of the neutral forms observed reduces with increasing both the temperatures of the cold substrate and of the vapour prior to deposition, enabled to conclude that the observed (neutral form) $\rightarrow$ zwitterion conversion occurs in the solid state, to the therein most stable zwitterionic species, and that intermolecular interactions in this phase should play an important role in the mechanism of proton transfer.

\section{Introduction}

It is well known that in solutions of polar or protic solvents, as well as in the crystalline phases, simple amino acids occur as zwitterions. ${ }^{1-10}$ Glycine, for example, the simplest amino acid, may exist in three different crystalline varieties $(\alpha-, \beta$ - and $\gamma$ glycine) whose structures were determined by X-ray or neutron diffraction and found to be formed exclusively by zwitterions. ${ }^{11-14}$

In the gas phase, however, simple amino acids exist in their neutral (non ionic) form. ${ }^{15-23}$ Indeed, for glycine, it has been shown that the zwitterion does not even correspond to a minimum on the gas-phase potential energy surface. ${ }^{24,25}$ The neutral forms of simple amino acids do also correspond to the observed species of these molecules isolated in low-temperature inert matrices. ${ }^{15-18,23}$

In the case of $N, N$-dialkyl substituted amino acids, their substantially greater solubility in non-polar solvents (e.g., benzene, carbon tetrachloride) enabled to examine the zwitterion vs. neutral form equilibrium in such solutions and evidence was pointed out of dominance of the neutral form under these experimental conditions. ${ }^{26-30} \mathrm{~A}$ few members of this family of amino acids, having moderately long alkyl carbon chains, have been found to present much lower melting points than expected for species having the zwitterionic character suggested by their room-temperature infrared spectra (e.g., $\beta$-( $N, N$-di- $n$-butylamino)butyric acid: $\left.{ }^{30} \mathrm{mp} 59^{\circ} \mathrm{C}\right)$. Contrarily to simpler amino acids, these compounds melt without substantial decomposition, and it was observed that in such systems, immediately before melting zwitterions convert partially to neutral forms, which could then be detected spectroscopically. ${ }^{30}$ These results were interpreted assuming that dipole electric fields in the crystals are the main factor responsible for stabilization of the zwitterionic structures of amino acids and that for the specific type of amino acids considered both zwitterions and neutral forms are true minima, with relative energies changing with temperature, the zwitterions being favoured by a low temperature. ${ }^{30}$ For simple amino acids, observation of neutral forms in solid state was never reported.

Taking into consideration that, as described, in the gas phase simple amino acids can be obtained in their neutral form, we decided to exploit the possibility of trapping this species in the solid state prepared by fast deposition of a pure gaseous amino acid sample onto a suitable substrate kept at very low temperature. Could the neutral forms of simple amino acids exist in the solid state pure compound?

In this work, glycine and the $N$-methylated derivatives, sarcosine ( $N$-methylglycine) and DMG ( $N, N$-dimethylglycine) were studied. These compounds are well known by their biochemical relevance, acting as cryo- and osmoprotectants ${ }^{31-33}$ and inhibitory neurotransmitters, ${ }^{34}$ and are also essential intermediary metabolites in several fundamental biological processes. ${ }^{35}$ The structures and vibrational spectra of the neutral forms of these amino acids have been recently studied in detail in our laboratory by matrix-isolation FTIR spectroscopy supported by extensive quantum chemical MO calculations. $^{15,17,23}$ The series of molecules considered in the present study enable us also to evaluate the importance of alkyl substitution on the nitrogen atom to the problem under analysis. 


\section{Materials and methods}

Glycine was obtained from Riedel-de Haën (purity 99.5\%). DMG and sarcosine were obtained from Aldrich (purity 99\%).

The infrared (IR) spectra were collected on a Mattson (Infinity 60AR Series) Fourier Transform infrared spectrometer equipped with a deuterated triglycine sulfate (DTGS) detector and a $\mathrm{Ge} / \mathrm{KBr}$ beamsplitter, with $0.5 \mathrm{~cm}^{-1}$ spectral resolution. Necessary modifications of the sample compartment of the spectrometer were made in order to accommodate the cryostat head and allow efficient purging of the instrument by a stream of dry air to remove water and $\mathrm{CO}_{2}$ vapours.

The low-temperature solid layers were prepared by fast deposition of the sublimated vapours of the compounds onto a CsI substrate which was previously cool down to $9 \mathrm{~K}$ by means of an APD Cryogenics close-cycle helium refrigeration system with a DE-202A expander. The compound to be studied was initially placed inside the cryostat, under high vacuum $\left(10^{-6} \mathrm{mbar}\right)$, in a specially designed sublimation mini-oven controlled by a DEW temperature controller. After depositing the compound, annealing experiments were performed by slowly increasing temperature up to $300 \mathrm{~K}$. IR spectra were collected during this process every 10 to $20 \mathrm{~K}$. In order to check for the reversibility of the observed phenomena, after the temperature exceeded $300 \mathrm{~K}$, the substrate was cooled back to $9 \mathrm{~K}$. The cooling cycle was also monitored spectroscopically by recording IR spectra each $10-20 \mathrm{~K}$ of temperature decrease.

The room-temperature IR spectra of the studied compounds as $\mathrm{KBr}$ pellets were also obtained for comparison with the thin layer data obtained at low temperature.

The Density Functional Theory (DFT) calculations were performed with the Gaussian 98 (revision A.9) program, ${ }^{36}$ using the $6-311++\mathrm{G}(\mathrm{d}, \mathrm{p})$ basis set $\mathrm{s}^{37}$ and the three-parameter density functional abbreviated as B3LYP, which includes Becke's gradient exchange correction ${ }^{38}$ the Lee, Yang, Parr correlation functional $^{39}$ and the Vosko, Wilk and Nusair correlation functional. ${ }^{40}$

Geometries were optimized using the Geometry Direct Inversion of the Invariant Subspace (GDIIS) method. ${ }^{41}$ Vibrational frequencies were calculated at the same level of theory and the nature of the critical points on the potential energy surface resulting from optimisation was determined by inspection of the corresponding calculated Hessian matrix. All optimised structures were confirmed to be minimum energy structures. The calculated frequencies were scaled down by a single factor (0.978) to correct them for the effects of basis set limitations, neglected part of electron correlation and anharmonicity effects. Normal coordinates analyses were undertaken in the internal coordinates space as described by Schachtschneider ${ }^{42}$ using the program BALGA and the optimised geometries and harmonic force constants resulting from the DFT(B3LYP)/6-311++G(d,p) calculations.

\section{Results and discussion}

Glycine has been extensively studied both experimentally and theoretically in the gaseous phase as well as in condensed states (solution, solid phases); sarcosine and DMG have received much less attention.

The structure of crystalline glycine (in its three crystalline varieties $^{11-14}$ ) and sarcosine $e^{35}$ have been reported. Vibrational data for glycine in the condensed phases include IR and Raman spectra obtained for the different crystalline varieties ${ }^{1,10}$ and in solution. ${ }^{6}$ Several isotopically substituted glycine analogues have been studied, ${ }^{6,8,10}$ as well as its alkaline and acid salts. ${ }^{1}$ To the best of our knowledge there were no detailed vibrational spectroscopic studies published so far dealing with sarcosine and DMG in the pure condensed phase.
Gas-phase structural studies on glycine include microwave spectroscopy and electron diffraction analysis. ${ }^{19-22}$ The low thermal stability of glycine precludes its IR study in the gas phase because it is not possible to obtain a concentration of the compound that is sufficient to register the spectra. However, glycine has been studied by low-temperature matrix isolation infrared spectroscopy (MI-IR), which provided unequivocal information on the species present in the glycine vapour prior to deposition. ${ }^{16-18}$ Three different conformers of monomeric neutral glycine were found to exist in the gas phase and their vibrational signatures could be established in detail. ${ }^{16-18}$ DMG and sarcosine have been studied for the first time recently in our laboratory by the MI-IR technique. ${ }^{15,23}$ Very interestingly, DMG was shown to be a notable system exhibiting conformationally selective aggregation in $\mathrm{Xe}$ matrices. ${ }^{15}$

Glycine has also been extensively studied by theoretical methods. ${ }^{16-18,24,25,43}$ These studies support the experimental observations regarding the lack of stability of the zwitterionic species for isolated glycine. Theoretical studies on both sarcosine and DMG neutral species have also been reported, including recent studies from this laboratory. ${ }^{15,23,44,45}$

Fig. 1 shows the IR spectra obtained for glycine deposited from the vapour onto a CsI substrate at $9 \mathrm{~K}$; two spectra are presented (traces (b) and (c)), corresponding to two different sublimation temperatures $\left(87\right.$ and $64{ }^{\circ} \mathrm{C}$, for (b) and (c), respectively). These spectra are compared with those obtained for $\alpha$-glycine in a $\mathrm{KBr}$ pellet at room temperature (trace (a)) and neutral glycine isolated in an argon matrix ${ }^{17}$ (trace (d)). Fig. 2 summarizes the results of the annealing experiments undertaken with the sample corresponding to trace (b) in Fig. 1.

The spectra of the low-temperature film clearly reveal the presence of a mixture of the neutral (aggregated) and zwitterionic (as $\alpha$-crystalline variety) forms of glycine. The bands due to the zwitterion closely match the spectrum of $\alpha$-glycine crystal shown as trace (a) in Fig. 1; their assignment has been done previously ${ }^{1,6,10}$ and will not be discussed here again. The additional bands observed in traces (b) and (c) are associated with neutral glycine and appear at ca. $3365 \mathrm{~cm}^{-1}(\nu \mathrm{O}-\mathrm{H}), 3310$ $\mathrm{cm}^{-1}\left(\nu \mathrm{NH}_{2}\right.$ as); $3220 \mathrm{~cm}^{-1}\left(\nu \mathrm{NH}_{2} \mathrm{~s}\right), 1715 \mathrm{~cm}^{-1}(\nu \mathrm{C}=\mathrm{O})$, $1643 \mathrm{~cm}^{-1}\left(\delta \mathrm{NH}_{2}\right), 1247 \mathrm{~cm}^{-1}(\nu \mathrm{CO}+\delta \mathrm{COH}), 1157 \mathrm{~cm}^{-1}$ $(\nu \mathrm{CN}), 1010 \mathrm{~cm}^{-1}(\tau \mathrm{C}-\mathrm{O}), 938\left(\nu \mathrm{CC}+\omega \mathrm{NH}_{2}\right), 866\left(\omega \mathrm{NH}_{2}+\right.$ $\nu \mathrm{CC}), 666(\delta \mathrm{OCO})$ and $484 \mathrm{~cm}^{-1}(\delta \mathrm{CC}=\mathrm{O})$. All these bands decrease of intensity upon annealing to higher temperatures (see Fig. 2) and are absent in the spectrum of the room temperature $\alpha$-glycine sample. Most of these bands are asymmetric or show more than one component, as expected taking into consideration that three conformers of neutral glycine exist in the gas phase prior to deposition. ${ }^{17}$

It is also clear from the observed spectra that neutral glycine molecules exist as aggregated species. Indeed, $\nu \mathrm{O}-\mathrm{H}, \nu \mathrm{NH}_{2}$ as, $\nu \mathrm{NH}_{2} \mathrm{~s}$ and $\nu \mathrm{C}=\mathrm{O}$ give rise to bands appearing at lower frequencies than for the monomer (see Fig. 1), while $\nu \mathrm{CO}+\delta \mathrm{COH}$ and $\tau \mathrm{C}-\mathrm{O}$ are observed at considerably higher frequencies. However, when the calculated spectrum for the centrosymmetric carboxylic acid neutral glycine dimer is taken into consideration, the bands assigned to the neutral form fit nicely the experimental data (see Fig. 2(b) and Table 1). The assignment of those bands to neutral glycine is also supported by the fact they occur at nearly the same frequencies as those previously reported ${ }^{1}$ for the corresponding vibrations in the alkaline (for vibrations of the $\mathrm{NH}_{2}$ group) or acid (for vibrations of the COOH group) salts of glycine: $3439 \mathrm{~cm}^{-1}(\nu \mathrm{O}-$ $\mathrm{H}), 3325 \mathrm{~cm}^{-1}\left(\nu \mathrm{NH}_{2}\right.$ as); $3212 \mathrm{~cm}^{-1}\left(\nu \mathrm{NH}_{2} \mathrm{~s}\right), 1745 \mathrm{~cm}^{-1}$ $(\nu \mathrm{C}=\mathrm{O}), 1630 \mathrm{~cm}^{-1}\left(\delta \mathrm{NH}_{2}\right), 1254 / 1216 \mathrm{~cm}^{-1}(\nu \mathrm{CO}+\delta \mathrm{COH})$, $1069 \mathrm{~cm}^{-1}(\nu \mathrm{CN}), 1009\left(\nu \mathrm{CC}+\omega \mathrm{NH}_{2}\right), 865 / 827\left(\omega \mathrm{NH}_{2}+\right.$ $\nu \mathrm{CC}), 674 \mathrm{~cm}^{-1}(\delta \mathrm{OCO})$ and $496 \mathrm{~cm}^{-1}(\delta \mathrm{CC}=\mathrm{O})$ (considering the results shown in Fig. 2, the assignment of the $\tau \mathrm{C}-\mathrm{O}$ vibration in the glycine acid salt to the band at 


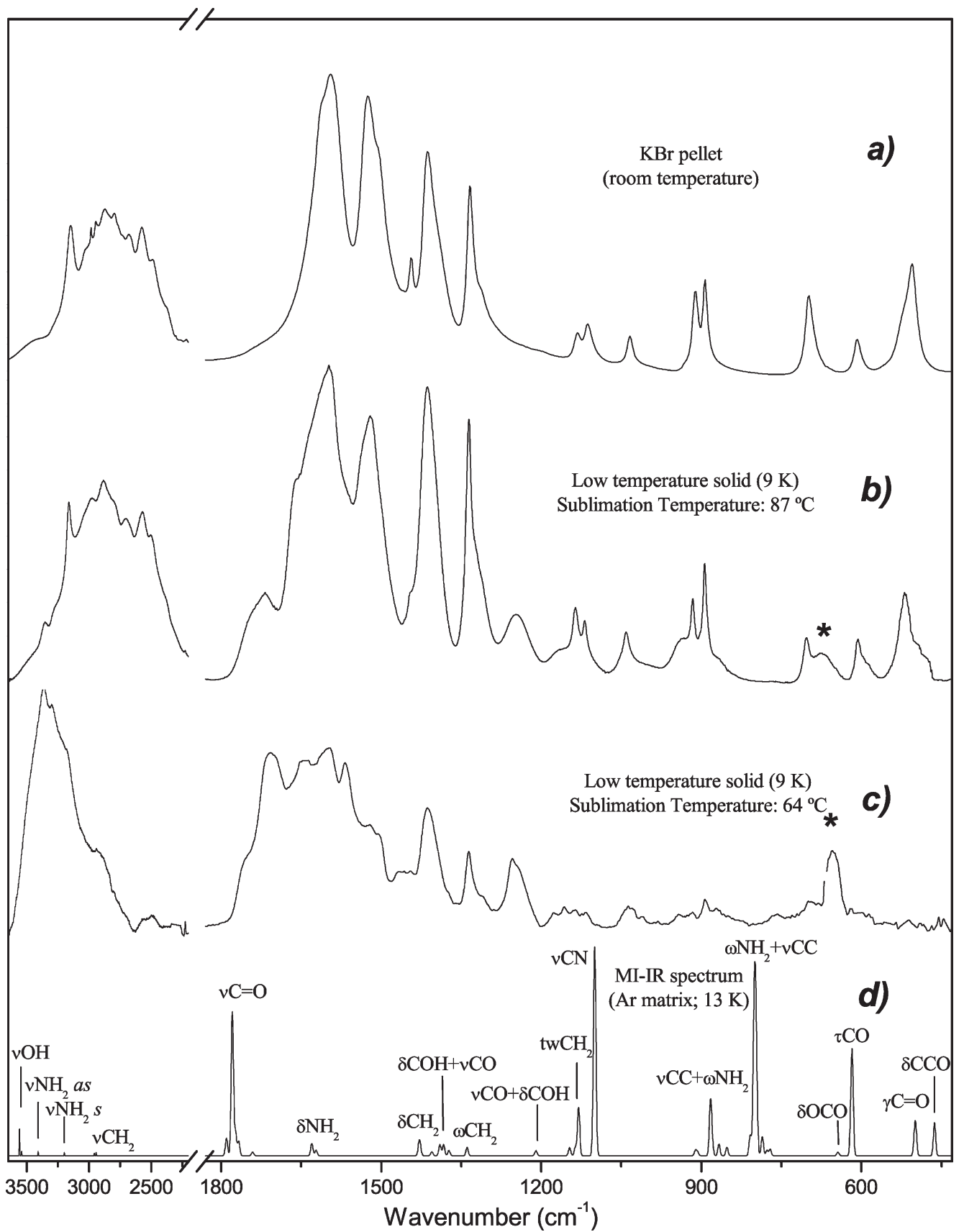

Fig. 1 IR spectra of glycine: (a) as a $\mathrm{KBr}$ pellet at room temperature; (b) and (c) low-temperature film at $9 \mathrm{~K}$ deposited from the sublimated vapour at 87 and $64^{\circ} \mathrm{C}$, respectively; (d) simulated MI-IR spectrum of argon isolated (13 K) monomeric form generated using the data shown in Table 5 of ref. 17. ${ }^{*} \mathrm{CO}_{2}$ band. In (d), only the relevant bands for discussion in the text are marked.

$804 \mathrm{~cm}^{-1}$ made in ref. 1 on the basis of relatively modest Hartree-Fock 6-31G(d) MO calculations does not appear to be correct).

All the bands ascribable to neutral glycine appear considerably more intense in the spectrum of the solid film obtained from deposition of the vapour at the lower sublimation temperature used (compare traces (b) and (c) of Fig. 1). This result can be easily understood considering local heating effects: when the hot glycine molecules land on the cooled substrate surface the thermal energy can be used to promote the proton shift converting the neutral species into the most stable (in the solid) zwitterions. The higher the temperature of the vapour is, the more important the local heating is, leading to a more extensive conversion of the neutral to zwitterionic species. It can also be noticed that, when the amount of neutral glycine in the film is larger, the degree of crystallinity of the sample reduces. Indeed, as mentioned before, the spectrum profile associated with the zwitterionic glycine shown in trace (b) of Fig. 1 (which corresponds to the sample obtained from the hotter vapour) matches almost perfectly that of $\alpha$-crystalline glycine, while the spectrum obtained from the cooler vapour (trace (c) in Fig. 1) shows noticeable differences when compared with the spectrum of the $\alpha$-crystal.

Another interesting question that could be elucidated by the present study concerns the problem of knowing where the proton transfer leading to the neutral $\rightarrow$ zwitterion or zwitterion $\rightarrow$ neutral glycine tautomerization occurs during sublimation. As mentioned before, the zwitterionic form of glycine does not correspond to a stable species for the isolated molecule. ${ }^{24,25}$ Polarization effects induced by the crystal upon the neutral glycine molecules approaching its surface can lead to a stabilization of the zwitterions. However, it is unlikely that this stabilization can be enough to reduce to a reasonable value the barrier for intramolecular proton transfer (which for 

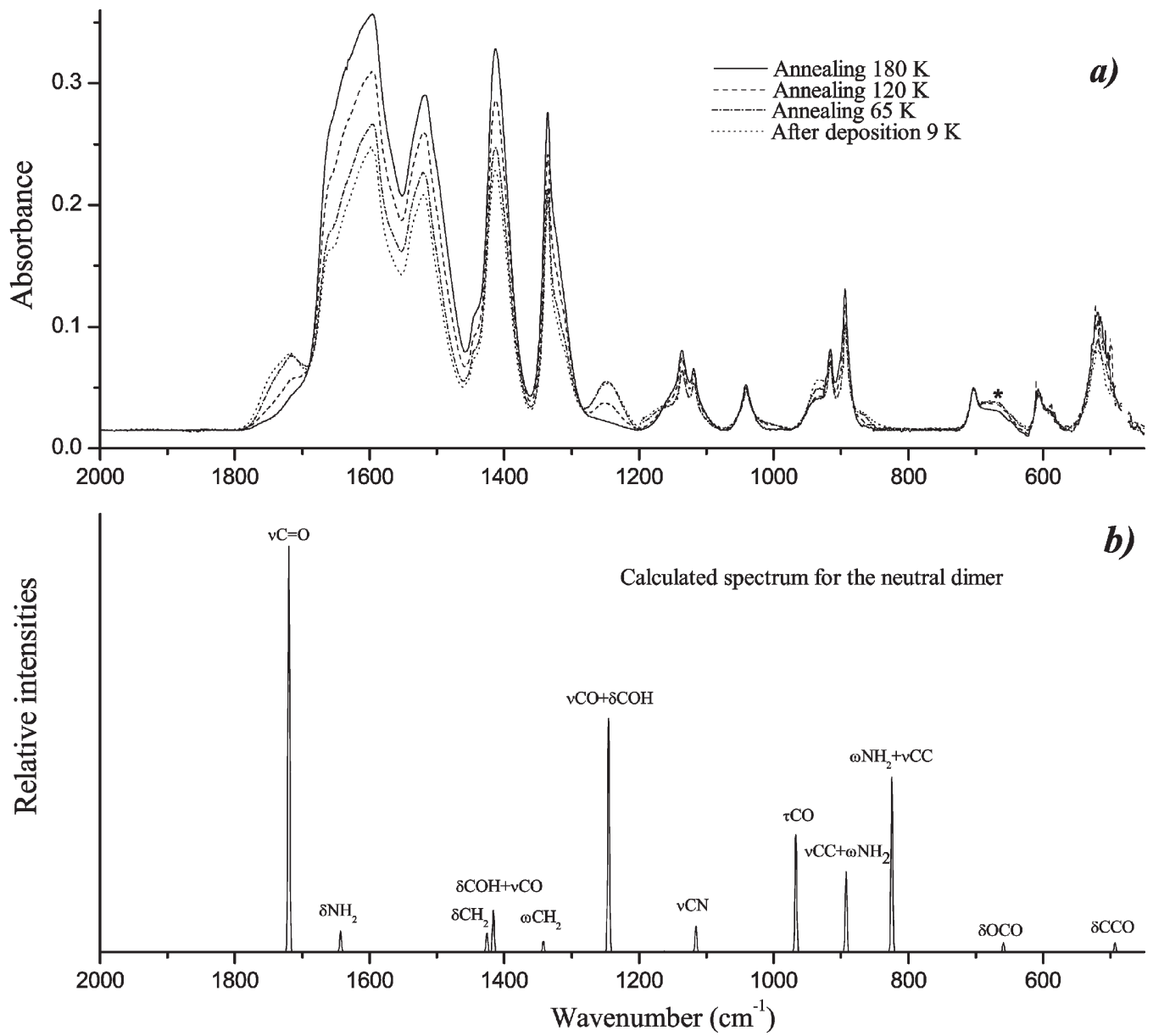

Fig. 2 (a) Annealing experiments carried out on the glycine low-temperature solid film. (b) 6-311++G(d,p) IR calculated spectrum of glycine dimer (scale factor: $0.978 ; ;^{15,23}$ centrosymmetric carboxylic acid dimer of neutral glycine with monomeric units in their most stable conformation ${ }^{17}$ ). ${ }^{*} \mathrm{CO}_{2}$ band.

glycine in the vacuum was found ${ }^{4}$ to be $c a .75 \mathrm{~kJ} \mathrm{~mol}^{-1}$ ), in order to enable the proton transfer to take place in the gas phase. On the other hand, the observed increase in the relative amount of the zwitterion in the solid phase with the sublimation temperature is consistent with the occurrence of the proton transfer in the solid phase, immediately after the landing of the molecules on the surface of the cold substrate, since a higher temperature of the vapour being deposited correlates with a more important local heating. Moreover, the observation of aggregates of neutral glycine in the solid state does also demonstrate the involvement of these species in the proton transfer, pointing also to the importance of the intermolecular forces within the crystal to this process. It is important to note that these conclusions are in consonance with the observation of Nash and coworkers that, for a series of $N$-dialkyl substituted amino acids, proton transfer in the solid phase leading to zwitterion $\rightarrow$ neutral tautomerization occurs prior to the melting of the compounds. ${ }^{26,27}$

The main conclusions obtained in this study from the experiments undertaken on glycine were also reinforced by additional experiments carried out on sarcosine and DMG. Figs. 3 and 4 summarize the results obtained for these two compounds. Assignments of the bands due to the neutral form are provided in Table 1 .

As seen in these figures, fast deposition of the vapour of these substances onto the cold substrate of the cryostat (kept at $9 \mathrm{~K}$ ) does also enable observation of a mixture of neutral and zwitterionic species. In the case of DMG, the vibrational signature of the zwitterion closely resembles that of the room-temperature spectrum of the compound in a $\mathrm{KBr}$ pellet (see Fig. 4). For sarcosine, however, such agreement is not so close, the signature of the zwitterion in the low-temperature film showing noticeable differences from the spectrum of the compound in $\mathrm{KBr}$ at room temperature (see Fig. 3). In fact, we collect experimental evidence indicating that sarcosine shall exist in a number of different crystalline polymorphs $\dagger$. With all probability, the polymorph formed in the low-temperature film was different from that prevalent at room temperature. In any case, the main general spectroscopic features are still comparable and enabled a clear identification of the bands due to zwitterionic forms in the low-temperature spectrum. For both compounds, DMG and sarcosine, the assignment of the dominant bands due to zwitterions is straightforward and does not require much attention.

In the case of DMG, the bands due to the neutral form are observed at $c a .3360 \mathrm{~cm}^{-1}(\nu \mathrm{O}-\mathrm{H}), 1728 / 1681 \mathrm{~cm}^{-1}(\nu \mathrm{C}=\mathrm{O})$, $1232 \mathrm{~cm}^{-1}(\nu \mathrm{CO}+\delta \mathrm{COH}), 1155 \mathrm{~cm}^{-1}\left(\gamma \mathrm{CH}_{3}\right), 1098 \mathrm{~cm}^{-1}$ $(\nu \mathrm{CN}), 1046 \mathrm{~cm}^{-1}(\tau \mathrm{C}-\mathrm{O}), 866 \mathrm{~cm}^{-1}(\nu \mathrm{CC})$ and $621 \mathrm{~cm}^{-1}$ ( $\delta \mathrm{OCO})$, fitting well the calculated spectrum for the centrosymmetric carboxylic acid like dimer, as shown in Fig. 4. For monomeric neutral form of DMG isolated in Xe matrices the analogous vibrations were observed ${ }^{15}$ at $3536 \mathrm{~cm}^{-1}(\nu \mathrm{O}$ $\mathrm{H}), 1780 / 1772 / 1770 \mathrm{~cm}^{-1}(\nu \mathrm{C}=\mathrm{O}), 1115 / 1113 / 1112 \mathrm{~cm}^{-1}$ $(\nu \mathrm{CO}+\delta \mathrm{COH}), 1174 \mathrm{~cm}^{-1}(\nu \mathrm{CN}), 893 \mathrm{~cm}^{-1}(\tau \mathrm{C}-\mathrm{O}), 837$

$\dagger$ By submitting a solid film of sarcosine to a series of consecutive warming-cooling cycles within the temperature range $9-430 \mathrm{~K}$ and probing it by IR spectroscopy we noticed that different spectral profiles could be observed, which are consistent with different crystalline arrangements of the compound. DSC studies on this compound are now being running to characterize its different solid phases and phase transitions. 
Table 1 Observed and calculated [DFT (B3LYP) $/ 6-311++\mathrm{G}(\mathrm{d}, \mathrm{p})]$ wavenumbers (within the $1800-450 \mathrm{~cm}^{-1}$ spectral region) and intensities $(I)$ for neutral forms of glycine, sarcosine and $\mathrm{DMG}^{a}$

\begin{tabular}{|c|c|c|c|c|c|c|c|c|c|c|c|c|c|c|}
\hline \multicolumn{5}{|l|}{ Glycine } & \multicolumn{5}{|l|}{ Sarcosine } & \multicolumn{5}{|l|}{ DMG } \\
\hline \multirow[b]{2}{*}{ Band $^{b}$} & \multicolumn{2}{|c|}{ Calculated } & \multicolumn{2}{|c|}{ Observed } & \multirow[b]{2}{*}{ Band $^{b}$} & \multicolumn{2}{|c|}{ Calculated } & \multicolumn{2}{|c|}{ Observed } & \multirow[b]{2}{*}{ Band $^{b}$} & \multicolumn{2}{|c|}{ Calculated } & \multicolumn{2}{|l|}{ Observed } \\
\hline & $\nu$ & $I$ & $\nu$ & $I$ & & $\nu$ & $I$ & $\nu$ & $I$ & & $\nu$ & $I$ & $\nu$ & $I$ \\
\hline$\nu \mathrm{C}=\mathrm{O}$ & 1719.9 & 753.5 & 1715 & $\mathrm{~s}$ & $\nu \mathrm{C}=\mathrm{O}$ & 1716.5 & 673.7 & 1726 & $\mathrm{~s}$ & $\nu \mathrm{C}=\mathrm{O}$ & 1717.4 & 574.8 & $1728 / 1681$ & $\mathrm{~s}$ \\
\hline$\delta \mathrm{NH}_{2}$ & 1643.1 & 38.4 & 1643 & w, sh & $\delta \mathrm{NH}$ & 1494.5 & 19.4 & 1507 & w, sh & $\delta \mathrm{CH}_{3}$ as & 1493.7 & 35.3 & 1466 & $\mathrm{~m}$ \\
\hline$\delta \mathrm{CH}_{2}$ & 1425.9 & 35.1 & - & & $\delta \mathrm{CH}_{3}$ as & 1471.4 & 3.5 & - & & $\delta \mathrm{CH}_{3}$ as & 1479.8 & 8.6 & - & \\
\hline$\delta \mathrm{COH}+\nu \mathrm{CO}$ & 1416.2 & 78.1 & - & & $\delta \mathrm{CH}_{2}$ & 1458.5 & 25.3 & 1456 & $\mathrm{w}, \mathrm{sh}$ & $\delta \mathrm{CH}_{2}$ & 1456.8 & 41.2 & - & \\
\hline $\mathrm{twNH}_{2}$ & 1353.5 & 0.1 & - & & $\delta \mathrm{CH}_{3}$ as & 1432.8 & 16.3 & - & & $\delta \mathrm{CH}_{3}$ as & 1445.5 & 14.4 & - & \\
\hline$\omega \mathrm{CH}_{2}$ & 1342.0 & 20.0 & - & & $\delta \mathrm{CH}_{3} s$ & 1416.2 & 27.4 & - & & $\delta \mathrm{CH}_{3}$ as & 1441.9 & 6.2 & - & \\
\hline$\nu \mathrm{CO}+\delta \mathrm{COH}$ & 1245.2 & 438.2 & 1247 & $\mathrm{~s}$ & $\delta \mathrm{COH}+\nu \mathrm{CO}$ & 1414.0 & 75.5 & - & & $\delta \mathrm{CH}_{3} s$ & 1413.0 & 46.4 & - & \\
\hline $\mathrm{twCH}_{2}$ & 1162.8 & 1.4 & - & & $\omega \mathrm{CH}_{2}$ & 1337.2 & 34.8 & 1318 & w (?) & $\delta \mathrm{CH}_{3} s$ & 1409.5 & 1.1 & - & \\
\hline$\nu \mathrm{CN}$ & 1115.6 & 47.1 & 1157 & $\mathrm{~m}$ & $\mathrm{twCH}_{2}$ & 1283.8 & 19.0 & 1280 & w (?) & $\delta \mathrm{COH}+\nu \mathrm{CO}$ & 1406.1 & 54.7 & 1396 & $\mathrm{~m}, \mathrm{sh}$ \\
\hline$\tau \mathrm{CO}$ & 967.1 & 218.1 & 1010 & $\mathrm{~m}, \mathrm{br}$ & $\nu \mathrm{CO}+\delta \mathrm{COH}$ & 1245.4 & 414.6 & 1239 & $\mathrm{~s}$ & $\omega \mathrm{CH}_{2}$ & 1342.4 & 6.2 & - & \\
\hline$\gamma \mathrm{CH}_{2}$ & 899.0 & 0.7 & - & & $\nu \mathrm{CN}$ & 1176.9 & 81.3 & 1178 & $\mathrm{~m}$ & $\mathrm{twCH}_{2}$ & 1327.0 & 18.4 & - & \\
\hline$\nu \mathrm{CC}+\omega \mathrm{NH}_{2}$ & 892.6 & 148.2 & 938 & $\mathrm{~m}$ & $\nu \mathrm{C}\left(\mathrm{H}_{3}\right) \mathrm{N}$ & 1126.3 & 41.4 & 1118 & $\mathrm{~m}$ & $\gamma \mathrm{CH}_{3}$ & 1300.9 & 54.0 & 1289 & $\mathrm{w}, \mathrm{sh}$ \\
\hline$\omega \mathrm{NH}_{2}+\nu \mathrm{CC}$ & 824.6 & 323.0 & 866 & $\mathrm{~m}, \mathrm{br}$ & $\gamma \mathrm{CH}_{3}$ & 1124.5 & 4.0 & - & & $\nu \mathrm{CO}+\delta \mathrm{COH}$ & 1238.6 & 526.7 & 1232 & $\mathrm{~s}$ \\
\hline$\delta \mathrm{OCO}$ & 659.1 & 17.2 & 666 & $\mathrm{w}$ & $\gamma \mathrm{CH}_{3}$ & 978.5 & 20.1 & - & & $\gamma \mathrm{CH}_{3}$ & 1169.5 & 15.1 & 1155 & $\mathrm{w}$ \\
\hline$\gamma \mathrm{CO}$ & 546.8 & 0.7 & - & & $\tau \mathrm{CO}$ & 969.3 & 192.9 & 987 & sh & $\nu \mathrm{C}\left(\mathrm{H}_{3}\right) \mathrm{N}$ as & 1149.0 & 7.1 & - & \\
\hline \multirow[t]{9}{*}{$\delta \mathrm{CCO}$} & 493.3 & 17.1 & 484 & $\mathrm{w}$ & $\gamma \mathrm{CH}_{2}$ & 929.1 & 16.0 & 941 & $\mathrm{w}$ & $\gamma \mathrm{CH}_{3}$ & 1088.7 & 10.9 & - & \\
\hline & & & & & $\nu \mathrm{CC}$ & 868.5 & 12.3 & - & & $\nu \mathrm{CN}$ & 1066.6 & 129.8 & 1098 & $\mathrm{~m}$ \\
\hline & & & & & $\gamma \mathrm{NH}$ & 733.2 & 316.9 & $\sim 809$ & $\mathrm{~m}, \mathrm{br}$ & $\gamma \mathrm{CH}_{3}$ & 1047.9 & 23.6 & 1056 & sh \\
\hline & & & & & $\delta \mathrm{OCO}$ & 664.0 & 21.8 & 670 & w (?) & $\tau \mathrm{CO}$ & 963.4 & 208.5 & 1046 & $\mathrm{~m}$ \\
\hline & & & & & $\gamma \mathrm{CO}$ & 547.3 & 7.8 & 546 & w & $\gamma \mathrm{CH}_{2}$ & 919.2 & 0.6 & - & \\
\hline & & & & & $\delta \mathrm{CCO}$ & 500.6 & 19.6 & - & & $\nu \mathrm{CC}$ & 859.9 & 9.8 & 866 & $\mathrm{~m}$ \\
\hline & & & & & & & & & & $\nu \mathrm{C}\left(\mathrm{H}_{3}\right) \mathrm{N} s$ & 829.2 & 3.8 & - & \\
\hline & & & & & & & & & & $\delta \mathrm{OCO}$ & 676.9 & 107.2 & 621 & $\mathrm{~m}$ \\
\hline & & & & & & & & & & $\delta \mathrm{CCO}$ & 502.3 & 16.4 & - & \\
\hline
\end{tabular}

${ }^{a}$ Wavenumbers in $\mathrm{cm}^{-1}$. Calculated wavenumbers refer to the carboxylic acid like dimer and were scaled by the factor 0.978 (see text). Calculated intensities in $\mathrm{km} \mathrm{mol}^{-1}$. Experimental intensities are relative intensities described qualitatively. $\nu$, stretching; $\delta$, bending; $\omega$, wagging; tw, twisting; $\gamma$, rocking; $\tau$, torsion; $\mathrm{s}$, strong; m, medium; w, weak; sh, shoulder; br, broad. (?) uncertain. ${ }^{b}$ Approximate description.

$\mathrm{cm}^{-1}(\nu \mathrm{CC})$ and $625 \mathrm{~cm}^{-1}(\delta \mathrm{OCO})$. Hence, as for glycine, comparison of the spectra of the low-temperature solid film and matrix isolation data for the monomer clearly indicates that in the solid film neutral DMG molecules exist as aggregated species. Indeed, $\nu \mathrm{O}-\mathrm{H}$ and $\nu \mathrm{C}=\mathrm{O}$ give rise to bands appearing at lower frequencies than for the monomer while $\nu \mathrm{CO}+\delta \mathrm{COH}$ and $\tau \mathrm{C}-\mathrm{O}$ are observed at higher frequencies, i.e., the usual pattern of frequency shifts upon aggregation is observed. Furthermore, in our previous studies on matrix isolated $\mathrm{DMG}$, we have studied in detail the aggregation process in Xe matrices. ${ }^{15}$ Noteworthy, the bands due to the observed matrix-isolated dimers of neutral DMG fit nicely $\ddagger$ the spectrum of neutral DMG now obtained.

For sarcosine, the bands ascribable to the neutral form are observed at $c a .3318 \mathrm{~cm}^{-1}(\nu \mathrm{O}-\mathrm{H}), 1726 \mathrm{~cm}^{-1}(\nu \mathrm{C}=\mathrm{O}), 1507$ $\mathrm{cm}^{-1}(\delta \mathrm{NH}), 1456 \mathrm{~cm}^{-1}\left(\delta \mathrm{CH}_{2}\right), 1239 \mathrm{~cm}^{-1}(\nu \mathrm{CO}+\delta \mathrm{COH})$, $1178 \mathrm{~cm}^{-1}(\nu \mathrm{CN}), 1118 \mathrm{~cm}^{-1}\left(\nu \mathrm{C}\left(\mathrm{H}_{3}\right) \mathrm{N}\right), 987 \mathrm{~cm}^{-1}(\tau \mathrm{C}-\mathrm{O})$, $941 \mathrm{~cm}^{-1}\left(\gamma \mathrm{CH}_{2}\right), \approx 809 \mathrm{~cm}^{-1}(\gamma \mathrm{NH})$ and $546 \mathrm{~cm}^{-1}(\gamma \mathrm{CO})$. As for glycine and DMG, the observed bands due to the neutral form of sarcosine fit well both the calculated spectrum for its centrosymmetric carboxylic acid like dimer (see Fig. 3) and for the dominant neutral form based aggregates observed for the compound isolated in argon. ${ }^{23}$

The comparison of the results obtained for the three amino acids in what concerns to the relative amount of neutral form that could be trapped at low temperature does also support the interpretation presented above for glycine regarding the importance of local heating effects. It shall be noticed that the conditions (temperature of the sublimation furnace and gaseous flux) at which the three compounds were deposited

$\ddagger$ The relevant bands due to DMG dimeric structures in Xe matrices appear at $1731 \mathrm{~cm}^{-1}(\nu \mathrm{C}=\mathrm{O}), 1231 \mathrm{~cm}^{-1}(\nu \mathrm{CO}+\delta \mathrm{COH}) ; 1171 \mathrm{~cm}^{-1}$ $\left(\gamma \mathrm{CH}_{3}\right) ; 1066 \mathrm{~cm}^{-1}(\nu \mathrm{CN}) ; 966 \mathrm{~cm}^{-1}(\tau \mathrm{C}-\mathrm{O}), 866 \mathrm{~cm}^{-1}(\nu \mathrm{CC})$ and $695 \mathrm{~cm}^{-1}\left(\right.$ (OCO). ${ }^{15}$ were not identical Indeed, they were essentially determined by the relative trends shown by the three compounds to be produced in the gaseous phase (without decomposition). DMG, sarcosine and glycine have melting points (at normal pressure) of $180.8,208.0$ and $255.4{ }^{\circ} \mathrm{C}$, respectively. DMG was also found in our experiments to be the more volatile compound and glycine the less volatile one, i.e., to produce identical fluxes of the gaseous compounds inside the cryostat, in our experiments the temperature of the furnace used increased in the order DMG $\left(45^{\circ} \mathrm{C}\right)$, sarcosine $\left(57^{\circ} \mathrm{C}\right)$, glycine $\left(87^{\circ} \mathrm{C}\right)$. Using these experimental conditions, the fluxes of deposition of all the three compounds were $c a \cdot 10^{-3}$ absorbance units of the $\nu \mathrm{C}=\mathrm{O}$ vibration per minute. As mentioned before, for glycine we have also used a considerably lower sublimation temperature $\left(64^{\circ} \mathrm{C}\right)$, leading to a flux of gaseous compound of $c a$. $10^{-4}$ (in the same units) and, as discussed above, to a considerably amount of trapped neutral compound. We cannot precisely determine the neutral/zwitterion population ratio trapped in each experiment, since this would require the knowledge of the intrinsic intensity of a particular absorption band at different low temperatures for both, the neutral form and zwitterion. In spite of that, the relative percentages can be easily obtained. Taken as reference the amount of neutral glycine trapped when the lowest flux of deposition was used, the relative percentages of neutral form obtained for DMG, sarcosine and glycine deposited using a nearly equal flux were $72 \%, 53 \%$ and $25 \%$, respectively, correlating well with the temperature of the gaseous flux and, thus, with the extent of the local heating: the lower the temperature of the arriving gas, the larger the percentage of the trapped neutral molecules (or, in other words, of neutral molecules that did not convert to zwitterions). The relative percentage values were obtained in the following manner: firstly, the ratio of the $\nu \mathrm{C}=\mathrm{O}$ band (at $1643 \mathrm{~cm}^{-1}$ ) originated in the neutral amino acid in the reference spectrum $(9 \mathrm{~K}$ spectrum of glycine deposited at the 

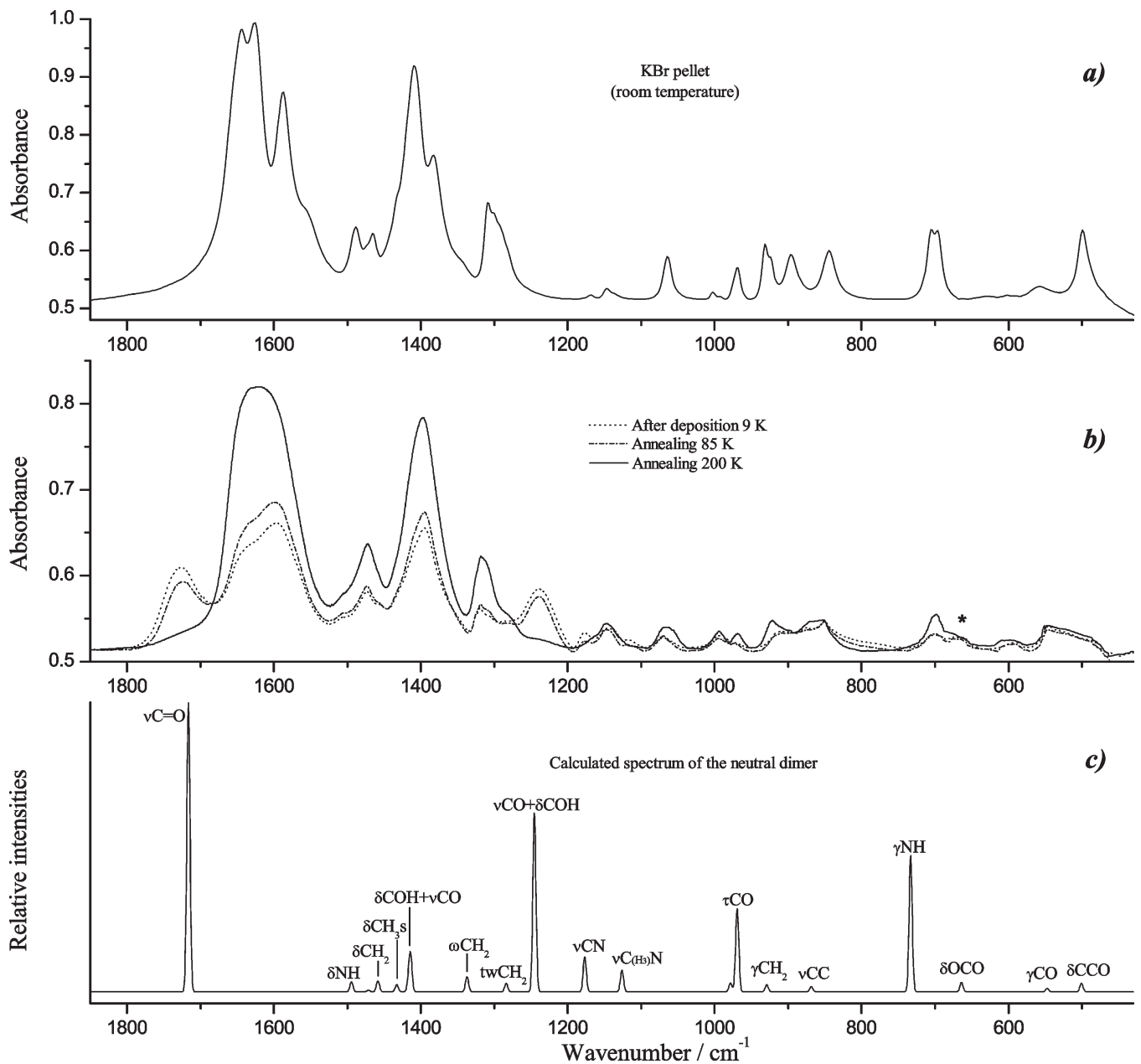

Fig. 3 (a) Annealing experiments carried out on the sarcosine low-temperature solid film. (b) 6-311++G(d,p) IR calculated spectrum of sarcosine dimer (scale factor: $0.978,3^{15,23}$ centrosymmetric carboxylic acid dimer of neutral sarcosine with monomeric units in their most stable conformation displaying an $\mathrm{O}=\mathrm{C}-\mathrm{O}-\mathrm{H}$ cis axis ${ }^{23}$ ). In (b), only the relevant bands for the discussion in the text are marked.

lowest flux) to the $\nu \mathrm{COO}$ sym carboxylate band (at 1413 $\mathrm{cm}^{-1}$ ) due to the zwitterion was measured (these bands correspond to features whose intensity is easy to measure accurately and are unequivocally ascribable to a single species); the equivalent intensity ratios are then obtained for the $9 \mathrm{~K}$ spectra of the samples of each amino acid prepared using the higher gaseous flux; finally, these last were divided by the reference intensity ratio.

The temperature of total conversion of the neutral form to the zwitterion depends also on the compound studied, being $275 \mathrm{~K}$ for $\mathrm{DMG}, 200 \mathrm{~K}$ for sarcosine and $180 \mathrm{~K}$ for glycine. This is in consonance with a greater stability of neutral $N$-alkyl substituted amino acids in solid state compared with the more ionic non- $N$-alkylated amino acids having higher melting points, as suggested by Parra-Mouchet et al. ${ }^{46}$ Finally, in order to obtain further support for the importance of local heating effects in determining the amount of neutral species trapped in the low-temperature solid films, we have also increased the temperature of the substrate and noticed that at a temperature near that at which after annealing of the film deposited at $9 \mathrm{~K}$ only zwitterions were observed, no neutral form could be trapped.

\section{Conclusions}

In this work, neutral forms of glycine, sarcosine and DMG were observed in the solid state pure compounds for the first time. For all the compounds studied, the spectra obtained at $9 \mathrm{~K}$, after fast deposition of the substance onto a cold substrate kept at that temperature, revealed the presence of both the neutral and zwitterionic amino acid forms. The signature of the neutral forms and their identification could be unequivocally established by taking into consideration the available data for the matrix-isolated neutral monomeric and aggregated amino acids ${ }^{15,17,23}$ as well as acid and alkaline salts of glycine. ${ }^{1}$ The amount of neutral form of the amino acids trapped in the low-temperature films was found to be essentially determined by local heating effects, which, in turn, depend upon the experimental conditions (e.g., flux intensity and temperature of the vapour being deposited): the more important the local heating the smaller the amount of neutral amino acid observed. Upon temperature increase, neutral forms convert non-reversibly to the zwitterionic forms. Being a measure of the relative stability of the neutral form in the solid state, the temperature of total conversion of the neutral forms to the zwitterions was found to increase with the number of methyl substituents at the nitrogen atom (DMG $>$ sarcosine $>$ glycine), as it could be expected. ${ }^{46}$ The whole set of data here obtained indicates that, with all probability, during deposition of the vapour of the compounds, the neutral form first aggregates and then uses the available energy to irreversibly convert, in the solid state, to the therein most stable zwitterionic species.

\section{Acknowledgements}

This work was supported by the Portuguese Fundação para a Ciência e a Tecnologia (Research Project POCTI/QUI/43366/ 2001 and Grant FCT \#SFRH/BPD/11499/2002). 

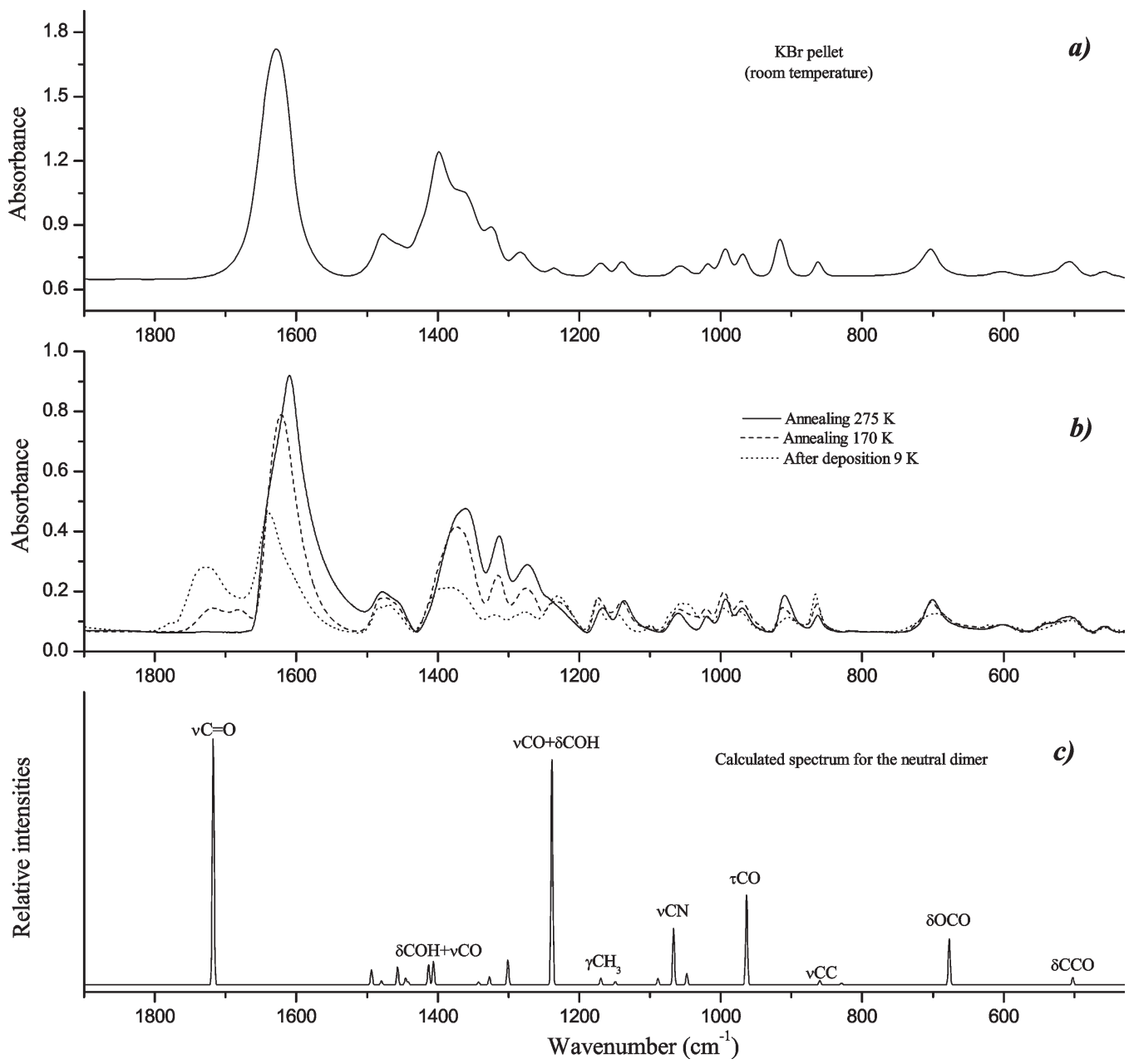

Fig. 4 (a) Annealing experiments carried out on the DMG low-temperature solid film. (b) 6-311++G(d,p) IR calculated spectrum of DMG dimer (scale factor: $0.978^{15,23}$ centrosymmetric carboxylic acid dimer of neutral DMG with monomeric units in their most stable conformation displaying an $\mathrm{O}=\mathrm{C}-\mathrm{O}-\mathrm{H}$ cis axis ${ }^{15}$ ). In (b), only the relevant bands for the discussion in the text are marked.

\section{References}

1 M. T. Rosado, M. L. T. S. Duarte and R. Fausto, Vib. Spectrosc., 1998, 16, 35

2 M. T. Rosado, M. L. T. S. Duarte and R. Fausto, J. Mol. Struct., 1997, 410-411, 343.

3 Y. Ding and K. Krogh-Jespersen, J. Comput. Chem., 1996, 17, 338.

4 J. Jensen and M. J. Gordon, J. Am. Chem. Soc., 1995, 117, 8159.

5 R. W. Williams, V. F. Kalasinsky and A. H. Lowrey, J. Mol. Struct., 1993, 281, 157.

6 K. Furic, V. Mohacek, M. Bonifacic and I. Stefanic, J. Mol. Struct., 1992, 267, 39.

7 T. C. Cheam, J. Mol. Struct., 1992, 274, 289.

8 S. F. A. Kettle, E. Lugwisha, J. Eckert and N. K. McGuire, Spectrochim. Acta., Part A, 1989, 45, 533.

9 R. Bonaccorsi, P. Palla and J. Tomasi, J. Am. Chem. Soc., 1984, 106, 1945.

10 C. Destrade, C. Garrigou-Lagrange and M.-T. Forel, J. Mol. Struct., 1971, 10, 203.

11 P. G. Jönsson and A. Kvick, Acta Crystallogr., Sect. B, 1972, 28, 1827.

12 R. E. Marsh, Acta Crystallogr., 1958, 11, 654

13 Y. Iitaka, Acta Crystallogr., 1960, 13, 35.

14 Y. Iitake, Acta Crystallogr., 1961, 14, 1.

15 A. Gómez-Zavaglia, I. D. Reva and R. Fausto, Phys. Chem. Chem. Phys., 2003, 5, 41.

16 S. G. Stepanian, I. D. Reva, E. D. Radchenko and L. Adamowicz, J. Phys. Chem. A, 2001, 105, 10664.

17 S. G. Stepanian, I. D. Reva, E. D. Radchenko, M. T. S. Rosado, M. L. T. S. Duarte, R. Fausto and L. Adamowicz, J. Phys. Chem., 1998, 102, 1041.

18 I. D. Reva, A. M. Plokhotnichenko, S. G. Stepanian, A. Yu. Ivanov, E. D. Radchenko, G. G. Sheina and Y. P. Blagoi, Chem.
Phys. Lett., 1995, 232, 141; I. D. Reva, A. M. Plokhotnichenko, S. G. Stepanian, A. Yu. Ivanov, E. D. Radchenko, G. G. Sheina and Y. P. Blagoi, Chem. Phys. Lett., 1995, 235, 617.

19 P. D. Godfrey and R. D. Brown, J. Am. Chem. Soc., 1995, 117, 2019.

20 K. Iijima and K. Tanaka, J. Mol. Struct., 1991, 246, 257.

21 (a) R. D. Sueram and F. J. Lovas, J. Am. Chem. Soc., 1980, 102, 7180; (b) L. Shäefer, H. Sellers, F. J. Lovas and R. D. Sueram, J. Am. Chem. Soc., 1980, 102, 6566.

22 R. D. Brown, P. D. Godfrey, J. W. V. Storey and M.-P. Bassez, J. Chem. Soc., Chem. Comm., 1978, 547.

23 A. Gómez-Zavaglia and R. Fausto, Vib. Spectrosc., submitted.

24 J. H. Jensen and M. S. Gordon, J. Am. Chem. Soc., 1995, 117, 8159.

25 Y. Ding and K. Krogh-Jespersen, Chem. Phys. Lett., 1992, 199, 261.

26 D. A. Horsma and C. P. Nash, J. Phys. Chem., 1968, 72, 2351

27 J. W.-O. Tam and C. P. Nash, J. Phys. Chem., 1972, 76, 4033.

28 D. L. Hughes, J. J. Bergan and E. J. J. Grabowski, J. Org. Chem., 1986, 51, 2579.

29 M. A. Peterson and C. P. Nash, J. Phys. Chem., 1985, 89, 522.

30 J. Higgins, N. A. Hodges, C. J. Olliff and A. J. Phillips, J. Pharm. Pharmacol., 1987, 39, 577.

31 A. W. Lloyd, J. A. Baker, G. Smith, C. J. Olliff and K. J. Rutt, J. Pharm. Pharmacol., 1992, 44, 507.

32 T. J. Anchordoguy, A. S. Rudolph, J. F. Carpenter and J. H. Crowe, Cryobiology., 1987, 24, 324.

33 A. Tebecis and A. DiMaria, Brain Res., 1972, 40, 373.

34 R. Binzak, S. Wevers, Y. Moolenaar, W. Lee, J. Hwu, U. Poggi-Bach, H. Engelke, J. Hoard, J. Vockley and J. Vockley, Am. J. Hum. Genet., 2001, 68, 839.

35 A. Mostad and S. Natarajan, Acta Chem. Scand., 1989, 43, 1004

36 M. J. Frisch, G. W. Trucks, H. B. Schlegel, G. E. Scuseria, M. A. Robb, J. R. Cheeseman, V. G. Zakrzewski, J. A. Montgomery, 
Jr., R. E. Stratmann, J. C. Burant, S. Dapprich, J. M. Millam, A. D. Daniels, K. N. Kudin, M. C. Strain, O. Farkas, J. Tomasi, V. Barone, M. Cossi, R. Cammi, B. Mennucci, C. Pomelli, C. Adamo, S. Clifford, J. Ochterski, G. A. Petersson, P. Y. Ayala, Q. Cui, K. Morokuma, D. K. Malick, A. D. Rabuck, K. Raghavachari, J. B. Foresman, J. Cioslowski, J. V. Ortiz, B. B. Stefanov, G. Liu, A. Liashenko, P. Piskorz, I. Komaromi, R. Gomperts, R. L. Martin, D. J. Fox, T. Keith, M. A. Al-Laham, C. Y. Peng, A. Nanayakkara, C. Gonzalez, M. Challacombe, P. M. W. Gill, B. G. Johnson, W. Chen, M. W. Wong, J. L. Andres, M. Head-Gordon, E. S. Replogle and J. A. Pople, GAUSSIAN 98 (Revision A.9), Gaussian, Inc., Pittsburgh, PA, 1998.

37 M. J. Frisch, M. Head-Gordon and J. A. Pople, Chem. Phys. Lett., 1990, 166, 281

38 A. D. Becke, Phys. Rev. A, 1988, 38, 3098

39 C. T. Lee, W. T. Yang and R. G. Parr, Phys. Rev. B, 1988, 37, 785.

40 S. H. Vosko, L. Wilk and M Nusair, Can. J. Phys., 1980, 58, 1200.

41 P. Csaszar and P. Pulay, J. Mol. Struct. (THEOCHEM), 1984, 114, 31 .

42 J. H. Schachtschneider, Technical Report, Shell Development Co., Emeryville, CA, 1969.

43 (a) L. F. Pacios, O. Gálvez and P. C. Gómez, J. Phys. Chem., 2001, 105, 5232; (b) D. T. Nguyen, A. C. Scheiner, J. W. Andzelm,
S. Sirois, D. R. Salahub and A. T. Hagler, J. Comput. Chem., 1997, 13, 1609; (c) V. Barone, C. Adamo and F. Lelj, J. Chem. Phys., 1995, 102, 364; (d) C. H. Hu, M. Shen and H. F. Schäfer III, J. Am. Chem. Soc., 1993, 115, 2923; (e) A. G. Császár, J. Am. Chem. Soc., 1992, 114, 9568; $(f)$ M. Ramek, V. K. W. Cheng, R. F. Frey, S. Q. Newton and L. Schäfer, J. Mol. Struct. (THEOCHEM), 1995, 235, 1; (g) R. F. Frey, J. Coffin, S. Q. Newton, M. Ramek, V. K. W. Cheng, F. A. Momany and L. Schäfer, J. Am. Chem. Soc., 1992, 114, 9568; (h) J. H. Jensen and M. S. Gordon, J. Am. Chem. Soc., 1991, 113, 7917; (i) C. E. Dykstra, R. A. Chiles and M. D. Garrett, J. Comput. Chem., 1981, 2, 266; (j) H. L. Sellers and L. Schäfer, J. Am. Chem. Soc., 1978, 100, 7728; (k) L. Schäfer, H. L. Sellers, F. J. Lovas and R. D. Suenram, J. Am. Chem. Soc., 1980, 102, 6566; (l) S. Vishveshwara and J. A. Pople, J. Am. Chem. Soc., 1977, 99, 2422 .

44 A. Headley and S. Starnes, J. Mol. Struct. (THEOCHEM), 1996, 370, 147

45 A. Headley and S. Starnes, J. Mol. Struct. (THEOCHEM), 1998, 453, 247.

46 J. Parra-Mouchet, W. H. Flink and C. P. Nash, J. Phys. Chem., $1985,89,524$ 